\title{
From Atoms to Functional Nanomaterials: Structural Modifications as Observed Using Aberration-Corrected STEM
}

\author{
S. I. Sanchez, ${ }^{1 \star}$ L. F. Allard, ${ }^{2}$ M. T. Schaal, ${ }^{1}$ S. M. Tonnesen, ${ }^{1}$ Y. Le, ${ }^{1}$ S. A. Bradley, ${ }^{1}$ \\ P.L. Bogdan, ${ }^{1}$ and G. J. Gajda ${ }^{1}$ \\ ${ }^{1}$ Honeywell UOP, 50 E. Algonquin Rd., Des Plaines, IL 60016 \\ ${ }^{2}$ Oak Ridge National Laboratory, P.O. Box 2008 Oak Ridge, TN 37831
}

*Sergio.Sanchez5@Honeywell.com

\begin{abstract}
Aberration-corrected STEM has become a standard analytical technique in the field of nanoscience. As "designer materials" have become more in demand in academic circles, verification of a desired product makes atomic-resolutionanalysis mandatory. Industry currently faces the same trend where tailor-made materials are customized for a given application. Here we show several examples where quantifiable atomic-scale manipulation of nanomaterials can have a dramatic impact on structure and, by extension, functionality.
\end{abstract}

Keywords: aberration-corrected scanning transmission electron microscopy (STEM), Pt and Pt-Re nanoparticle catalysts, $\mathrm{WS}_{2}$ transition metal sulfide nanostructures, ex situ thermal processing, atomic resolution imaging

\section{Introduction}

For over a decade aberration-corrected scanning transmission electron microscopy (AC-STEM) has proven essential in the study of nanomaterials [1]. Nanomaterials are essentially assemblies of atoms arranged in such a way that they can serve important new applications. Considering their small size, it would not be surprising that subtle changes in the atomic structure can significantly impact functionality. For such systems, spherical AC-STEM employing a field-emission electron source is an essential tool for probing atomic-level structural modifications in nanomaterials.

The manner in which a collection of atoms assembles into a designed architecture is a critical topic. Equally important, however, is ensuring the generated structures fall within the nano-regime [2], a range of dimensional confinement in which materials exhibit non-bulk-like properties. There are two approaches to achieving this goal: top-down and bottom-up. The former generally requires the removal of material to the point where it is finely dispersed, relying heavily on physical alteration of the host material. Techniques toward this end are laser ablation, sputtering, and lithography, to name a few [3]. Challenges to this approach are the inability to finely control the formed structures, leading to polydispersity. Equally as important is the fact that not all nanomaterials are made of a single element so compositional control at atomic-length scales becomes important. Barring use of homogeneous multicomponent bulk systems for a desired product, compositional control poses a significant challenge for the top-down approach. Lastly, a major hurdle in this approach is finding the technologies necessary for commercial-scale production.

The bottom-up approach begins with a series of dispersed atoms and amasses them to form a desired architecture. This approach relies more on chemically driven responses toward nanoscale assemblies. Environmental factors, such as temperature, pressure, oxidizing or reducing conditions, and reagent concentration, can all be used to drive the modification of atomic structure [2]. There are several advantages to this approach, the first of which is the versatility in terms of the products that can be made. Particles of a certain composition, at an appropriate temperature and pressure, can reach a metastable nanostructural state. Employing thermodynamic concepts, one can encourage specific changes by modifying key environmental factors. By imposition of chemical factors (presence of $\mathrm{O}_{2}$, acids, etc.), one can further assemble a structure for a specific purpose. From an industrial standpoint such a result is highly desirable since it offers the possibility of customer-specific products and is more compatible with large-scale production of materials.

Current efforts aim to track functional nanostructure formation starting from dispersed atomic states. In this article we present a few case studies documenting how a modified atomic structure can impact functionality. The first case considers $\mathrm{Pt} / \gamma-\mathrm{Al}_{2} \mathrm{O}_{3}$, a widely used industrial catalyst, and examines the $\mathrm{H}_{2}$ reduction process by which the material is activated. Next this study is extended to a bimetallic system ( $\mathrm{Pt}-\mathrm{Re} / \gamma-\mathrm{Al}_{2} \mathrm{O}_{3}$ ) and evaluates the use of less pure $\mathrm{H}_{2}$ during the reduction process. Then transition metal sulfide nanostructures $\left(\mathrm{WS}_{2}\right)$ are analyzed to evaluate their development as atoms self-assemble to form ordered sheet-like nanostructures. Lastly, we impose a post-treatment on these sheets to modify their edge-structure.

\section{Materials and Methods}

Sample preparation. Samples were prepared by grinding to a powder as-received commercial catalyst pills $\left(\mathrm{Pt} / \gamma-\mathrm{Al}_{2} \mathrm{O}_{3}\right.$ and Pt-Re $/ \gamma-\mathrm{Al}_{2} \mathrm{O}_{3}$, separately). Powders were deposited directly on 200 mesh holey carbon Nylon grids (Pacific Grid Tech) for imaging and elemental analyses. The transition metal oxide/sulfide system $\left(\mathrm{WO}_{\mathrm{x}} \rightarrow \mathrm{WS}_{2}\right)$ was received in the form of a powder, which was embedded into an Epon epoxy matrix. After curing, the epoxy pellets were microtomed to $70-90 \mathrm{~nm}$ thin-sections and loaded onto holey carbon Nylon grids.

Methodology. $\mathrm{Pt} / \gamma-\mathrm{Al}_{2} \mathrm{O}_{3}$ in its inactive form (oxide) was loaded onto a Protochips Aduro thermal e-chip in situ specimen holder. The specimen holder was heated (via thermal e-chip) in an ex situ reactor to $500^{\circ} \mathrm{C}$ where the reactor supplied the flowing $\mathrm{H}_{2}$ for several time intervals $(5,15,30,60$, and 90 minutes). After each reduction period the sample in its holder was removed from the reactor and inserted into a microscope for analysis. After STEM analysis, the sample was re-inserted into the reactor for further reduction (Figure 1). Images and 


\section{Combining data \\ quality with \\ intuitive operation.}

ZEISS EVO

// RELIABILITY

MADE BY ZEISS

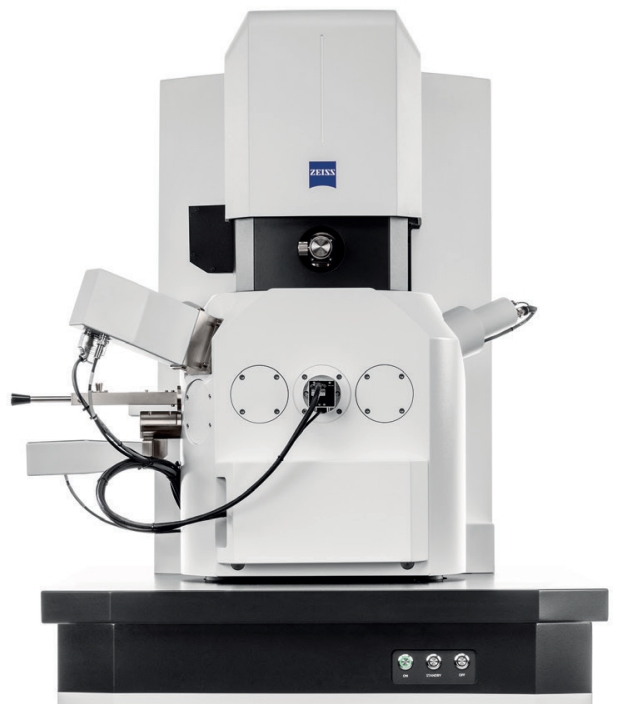

EVO 15

Your modular SEM platform for routine investigations and research applications

The instruments of the EVO family combine high performance scanning electron microscopy with an intuitive, user-friendly experience that appeals to both trained

microscopists and new users. With its comprehensive range of available options,

EVO can be tailored precisely to your requirements, whether you are in life sciences, material sciences, or routine industrial quality assurance and failure analysis. 


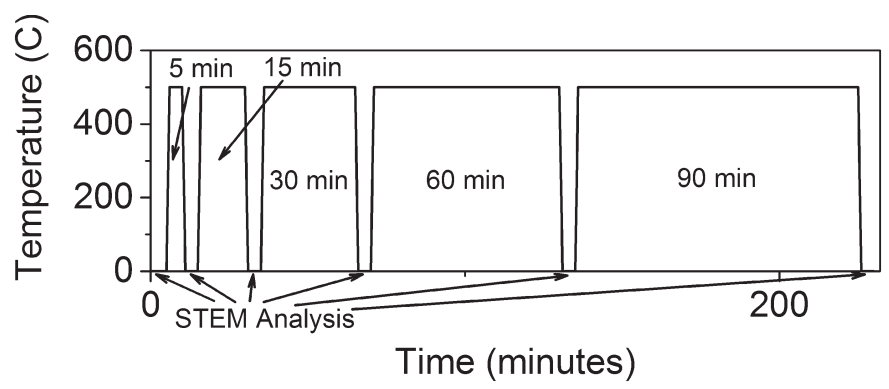

Figure 1: Temperature profile outlining reduction scheme used for the $\mathrm{Pt} / \gamma-\mathrm{Al}_{2} \mathrm{O}_{3}$ sample.

analyses were acquired from the same locations after each reduction step.

For the metal oxide/sulfide, the initial oxide powder was divided into four parts. One part was left as an oxide, and the other three were inserted into a sulfiding apparatus and sulfided up to different temperatures $\left(200^{\circ} \mathrm{C}, 310^{\circ} \mathrm{C}\right.$, and $\left.413^{\circ} \mathrm{C}\right)$. After the sulfidation treatment the samples were embedded, microtomed to thin-sections, and loaded into the microscope.

Electron microscopy. With the exception of the $\mathrm{Pt} / \gamma-\mathrm{Al}_{2} \mathrm{O}_{3}$ sample, which was examined in a $\mathrm{C}_{\mathrm{s}}$-corrected JEOL 2200FS at $200 \mathrm{keV}$ in STEM mode, all samples were examined in a $\mathrm{C}_{\mathrm{s}}$-corrected Titan 80-300 electron microscope equipped with ChemiSTEM $^{\mathrm{TM}}$ (FEI Company) technology. Imaging was conducted at $200 \mathrm{keV}$ in STEM mode using a Fischione model 3000 high-angle annular dark-field (HAADF) detector with inner and outer cutoff angles of 40 and 200 $\mathrm{mrad}$, respectively. The electron probe was focused to $0.8 \AA$, and the beam dosage at the specimen was $50 \mathrm{e} /$ $\AA^{2}$.s. Nanoparticle diameters were determined taking the average of every possible diameter that traverses the center of a particle between two tangent points. Atom, dimer, trimer, and particle counting was done manually for a series of images collected at the same magnification. Perimeter to area $(\mathrm{P} / \mathrm{A})$ ratio measurements for metal sulfide nanostructures were obtained by measuring the boundary lengths of individual nanostructures and integrating the area that was bound within.

The ChemiSTEM package comprises a high-brightness fieldemission source (X-FEG Schottky) for enhanced beam current and four $30 \mathrm{~mm}^{2}$ silicon drift detectors (SDDs) (Bruker Corp.) integrated into the objective lens for a total collection solid angle of 0.7 sr. Elemental analyses of individual nanoparticles was done by point analysis using a defocused electron probe to minimize beam damage. Collection times were no longer than $20 \mathrm{~s}$ to avoid beam damage. Both the $\mathrm{L} \alpha$ and $\mathrm{L} \beta \mathrm{X}$-ray lines were used for Pt and Re quantitative analysis.

\section{Results and Discussion}

$\mathrm{Pt} / \gamma-\mathrm{Al}_{2} \mathrm{O}_{3}$. Many nanomaterials contain a precious metal ( $\mathrm{Au}, \mathrm{Ag}, \mathrm{Pt}, \mathrm{Pd}$, etc.) in their formulation. The drawback associated with the use of $\mathrm{Pt}$ is the expense associated with it. Maximizing the dispersion of the metal (fraction of Pt atoms on the particle surface) then becomes important in order to offset its cost. The $\mathrm{Pt} / \gamma-\mathrm{Al}_{2} \mathrm{O}_{3}$ catalyst, used in the production of petrochemicals and transportation fuels, is a widely studied system. In its inactive state, the Pt is in the form of oxidized, dispersed, single $\mathrm{Pt}$ atoms bound by $\mathrm{O}$ atoms. The source of this $\mathrm{O}$ is from both air and the $\mathrm{O}$ atoms on the $\gamma-\mathrm{Al}_{2} \mathrm{O}_{3}$ support forming Pt-O bonds. Activation of the material is achieved by subjection to $\mathrm{H}_{2}$, which helps break the Pt-O interaction and reduces the $\mathrm{Pt}^{+\mathrm{x}}$ species to $\mathrm{Pt}^{0}[4]$.

To understand the reduction process, we used an ex situ approach: The fraction of $\mathrm{Pt}$ in the form of atoms (rather than clusters) was used to monitor completeness of reduction. Figures $2 \mathrm{a}$ and $2 \mathrm{~b}$ show the changes in the numbers of single atoms (small circles), dimers (squares), trimers (triangles), and clusters (large circles) over the course of reduction. These results were quantified manually from 20 images per sample. Figure $2 \mathrm{c}$ shows that the number of single atoms decreases and stabilizes after approximately $30 \mathrm{~min}$; whereas, the numbers of dimers and trimers remain unchanged. Figure $2 \mathrm{~d}$ shows that

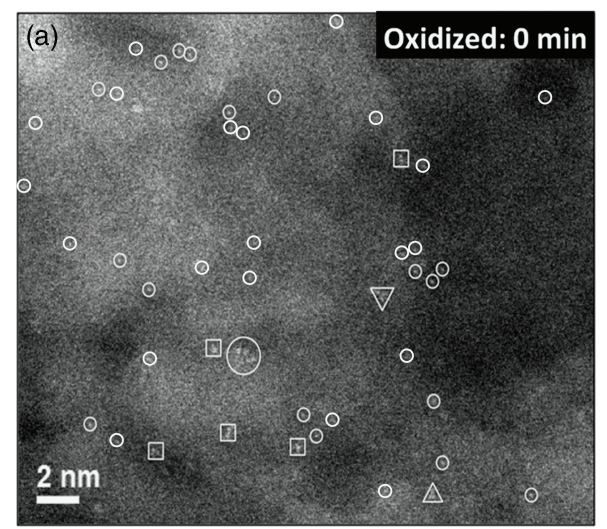

(c)

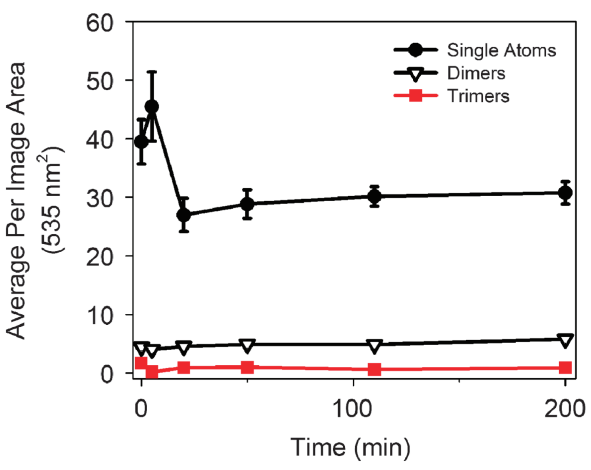

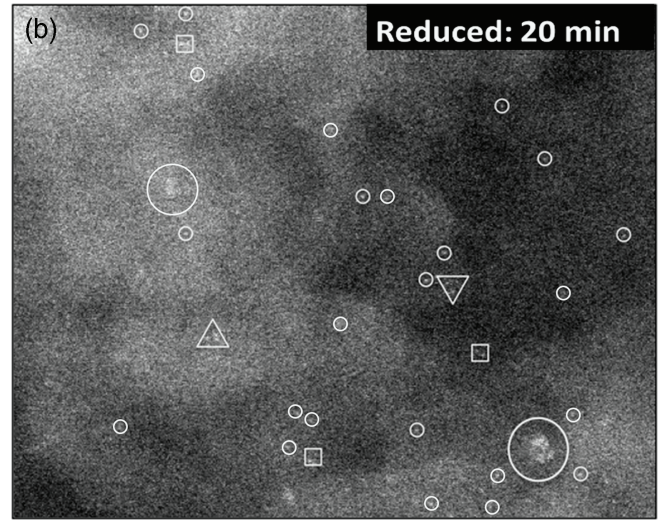

(d)

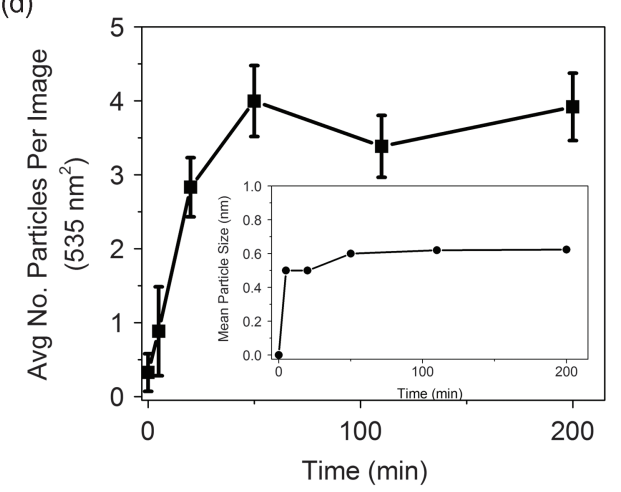

Figure 2: Atomic-resolution HAADF STEM images of $\mathrm{Pt} / \gamma-\mathrm{Al}_{2} \mathrm{O}_{3}$ in the (a) oxidized and (b) reduced (20 min) state. Small circles, to help guide the eye, indicate single atoms. (c) Abundance of single atoms, dimers, and trimers as a function of reduction time. The mean particle size monitored as a function time is shown in (d). Inset in (d) is the average number of particles per image relative to the reduction time. Scale bar in (a) is applicable to (b). 

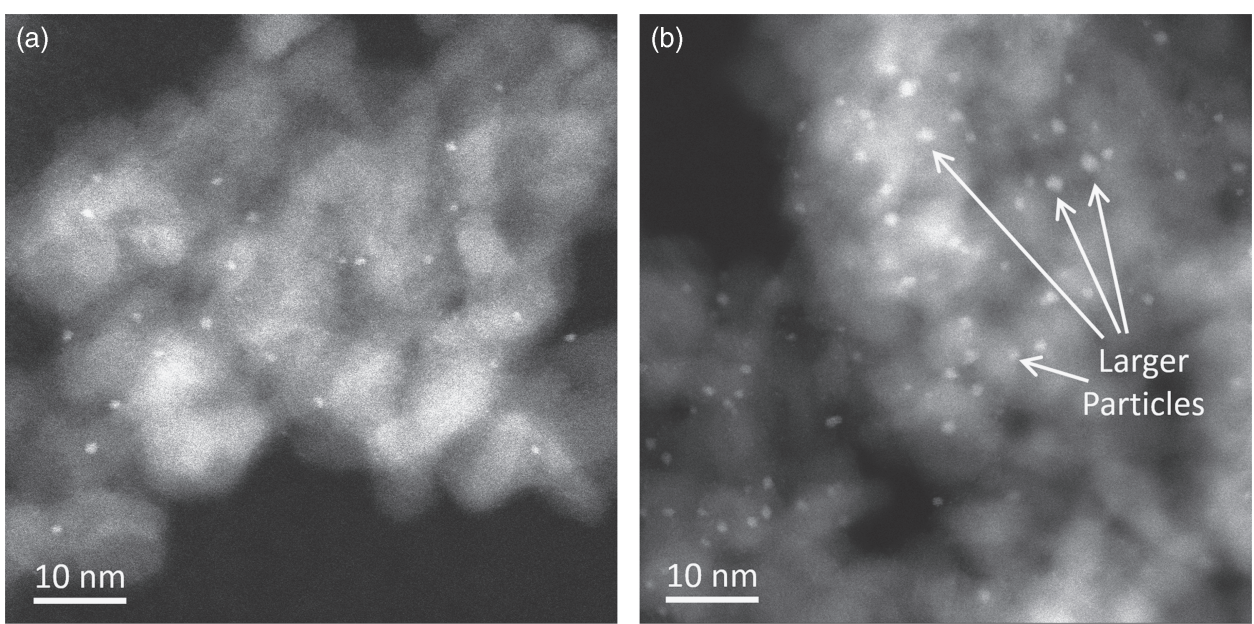

(c)

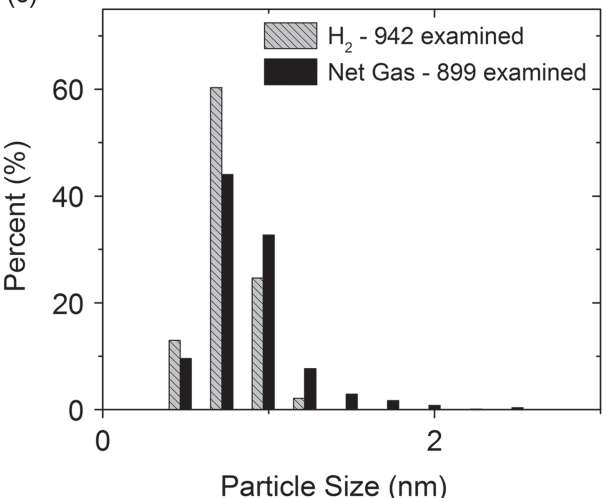

(d)

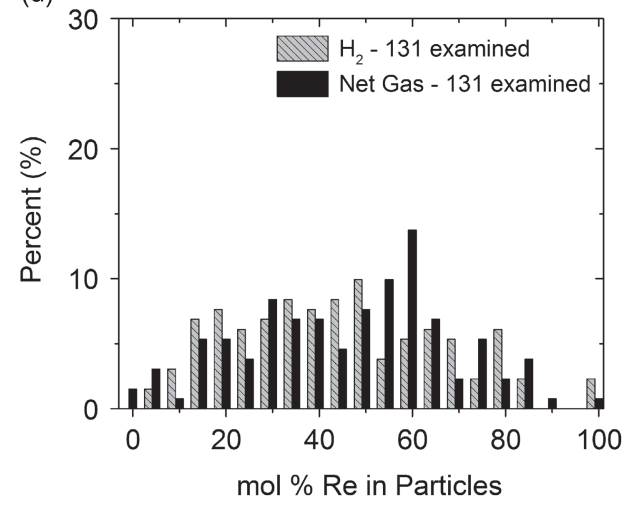

Figure 3: HAADF STEM images of Pt-Re/ $\gamma-\mathrm{Al}_{2} \mathrm{O}_{3}$ reduced via (a) $\mathrm{H}_{2}$ and (b) Net Gas. (c) and (d) show distribution histograms comparing (c) nanoparticle size and (d) composition based on the reduction treatments.

the average number of particles stabilized, as does the average particle size (inset), after the same amount of time. From these data it appears that a large fraction of the single atoms are responsible for forming the larger reduced Pt particles. This is important because it indicates that perhaps a way can be found to use less Pt in these formulations. Alternatively, these findings could be used to devise a method to maximize the effectiveness of the Pt present.

$\mathrm{Pt}-\mathrm{Re} / \gamma-\mathrm{Al}_{2} \mathrm{O}_{3}$. We extend these findings to a bimetallic Pt-Re system where the addition of the secondary metal yields synergistic effects-these being either electronic via charge-transfer, the generation of moieties by the dilution of Pt by secondary metal, or the presence of the second metal effectively imposing its chemistry $[5,6]$. As with $\mathrm{Pt} / \gamma-\mathrm{Al}_{2} \mathrm{O}_{3}$, activation of this material can be achieved via reduction in $\mathrm{H}_{2}$. Several costs have to be factored into the preparation of a catalyst, of which one is the use of high-purity $\mathrm{H}_{2}$ (g). A cost-saving technique practiced by some refiners utilizes Net Gas (a mixture of $\mathrm{H}_{2}$ and light hydrocarbon gases such as $\mathrm{CH}_{4}$, $\mathrm{C}_{2} \mathrm{H}_{6}$, CO, etc.) instead of high-purity $\mathrm{H}_{2}$ [6]. The effect of Net Gas reduction on reforming catalysts is not known, although changes to catalytic behavior have been observed [6]

Figure 3 shows a comparison of size distributions for the same batch of Pt-Re $/ \gamma-\mathrm{Al}_{2} \mathrm{O}_{3}$ catalyst: one reduced by the standard method using $\mathrm{H}_{2}$ and the other using Net Gas. With Net Gas reduction, the most obvious difference was the presence of larger particles dispersed throughout the sample.
This observation was reflected in the size-distribution histograms presented in Figure 3c. These results were reinforced at atomic resolution where the fraction of individual atoms of $\mathrm{Pt}$ and $\mathrm{Re}$ was higher for $\mathrm{H}_{2}$ reduction compared to Net Gas reduction (not shown). Compositional analysis of the metal sites, on the other hand, did not reveal significant differences between the two samples. Figure 3d shows broad distribution of compositions for individual particles in both samples with no obvious difference between them. The results indicate that the amount of Pt-Re interaction is equivalent regardless of the gas stream used during reduction. These data show a lower metal dispersion for the Net Gas treated sample without altering the composition of the metal particles. This would in all likelihood result in changes to catalytic performance. From a broader perspective this result stimulates interest as it opens doors toward performancebased customization of materials.

Metal sulfides $\left(\mathrm{WS}_{2}\right)$. Transition metal sulfides are used as lubricants, electrocatalysts, and as catalysts in oil and gas refining. Functionality as a lubricant comes from the graphenelike sheet structures these metal sulfides adopt, which can intercalate amongst each other [7]. As catalysts, the edges of these sheet-like structures provide undercoordinated environments that are widely regarded as reactive centers that promote chemical reactions $[8,9]$.

Given the minute changes that can give rise to significant differences in catalytic performance, it is instructive to understand the structural development of these metal sulfides. Figure 4 shows the growth of $\mathrm{WS}_{2}$ nanostructures as they form from $\mathrm{WO}_{\mathrm{x}}$ (room-temperature oxide), sulfided in $\mathrm{H}_{2} \mathrm{~S} / \mathrm{H}_{2}$ a gas stream at $413^{\circ} \mathrm{C}$, to become well-ordered, graphite-like sheets of $\mathrm{WS}_{2}$. Figure 4 shows the final product to be 2-dimensional sheets of $\mathrm{WS}_{2}$, where only $\mathrm{W}$ atoms are imaged under these conditions. The sheets appear to be terminated by faceted edges forming geometric-like shapes.

Since the edges of the sheets are considered to be the active sites $[8,9]$, it would make sense to maximize their frequency to advance performance. In the refining industry this $\mathrm{WS}_{2}$ phase is generally coupled with promoter species such as $\mathrm{Ni}$ (or Co) to improve activity [8-10]. Figure $5 \mathrm{a}$ is an atomicresolution HAADF-STEM image of a $\mathrm{WS}_{2}$-only sample where the $\mathrm{W}$ atoms are seen in a triangular morphology. Figure $5 \mathrm{~b}$ presents a Ni-promoted $\mathrm{WS}_{2}$ nanostructure where edge-sites have become populated with more discontinuities, consistent with literature reports $[8,9]$. It has been proposed that edge-site modification stems from incorporation of the promoter atoms into the edge-structure. This observation is consistent with 


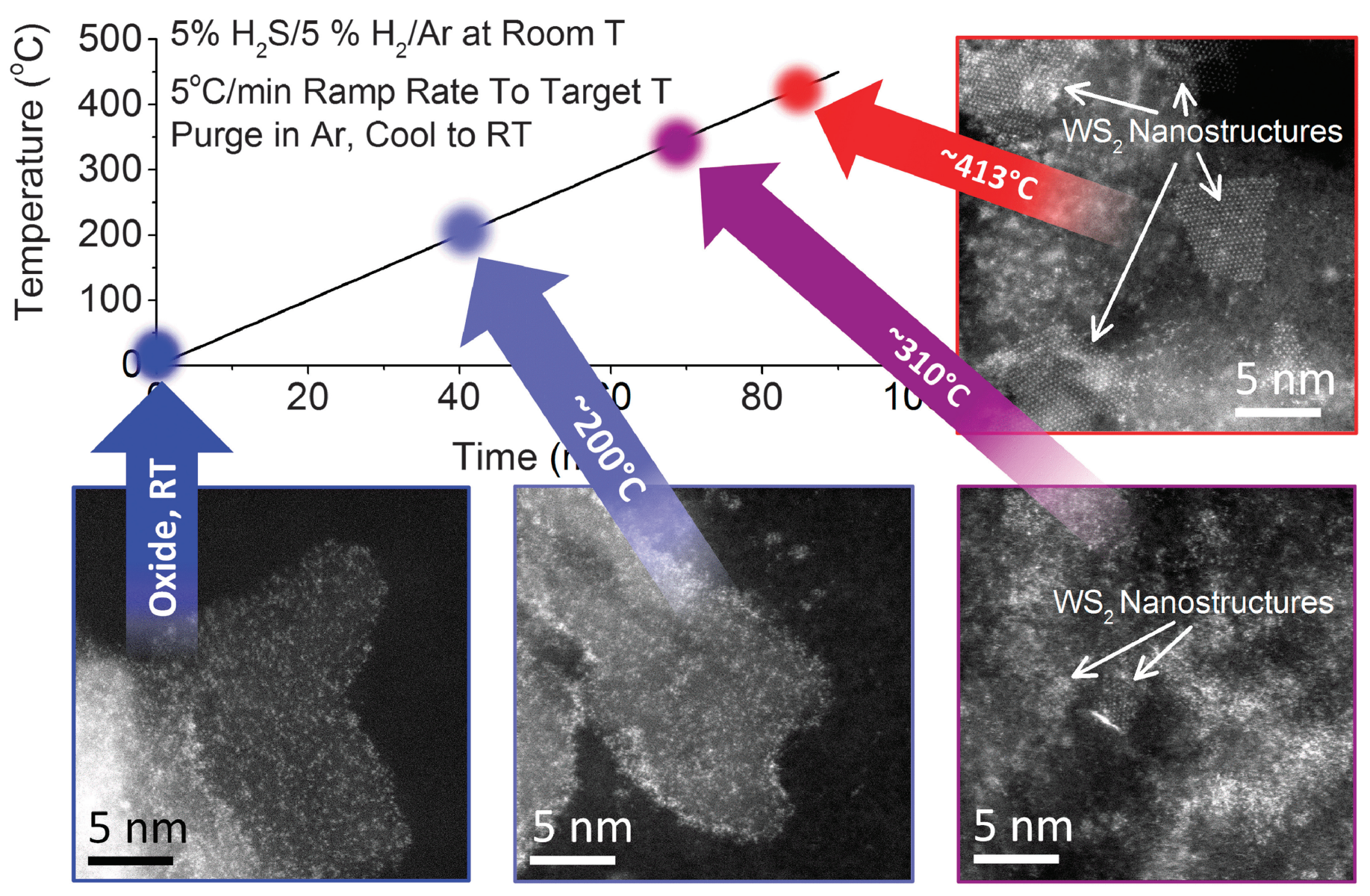

Figure 4: Atomic-resolution HAADF STEM images sampling $\mathrm{WS}_{2}$ nanostructure development during the ex situ sulfidation process at various temperatures. Arrows within images indicate locations of $\mathrm{WS}_{2}$ sheet-like structures.

electron energy loss spectrometry (EELS) work done by others $[11]$ and can serve as indirect confirmation that the promoter species also modifies the sheet edge-structure, which in turn gives rise to enhanced catalytic behavior.

To quantitatively understand the change in dispersion, the perimeter $(\mathrm{P})$ and area $(\mathrm{A})$ were measured for a series of individual $\mathrm{WS}_{2}$ sheets. Presumably, if edge-sites are the active component of these nanomaterials $[8,9]$, then it follows that an increased dispersion (a higher P/A ratio) would be beneficial to performance. Figure 5 shows that the Ni-promoted sample has a higher $\mathrm{P} / \mathrm{A}$ ratio indicative of more edge-sites. Literature reports observing the same structural phenomena [8, 9], coupled with internal testing results for these two catalysts, show clear improvements in yield and activity with promoter addition.

The next question is whether the performance benefits are chemical (associated more with the presence of a different elemental species) or physical (related to the structural modification) in nature. Toward this end, an attempt was made to understand whether a $\mathrm{W}$-only sample with comparable $\mathrm{P} / \mathrm{A}$ ratios to the $\mathrm{W}$-Ni system could achieve similar performance. The idea would be that oxidation of the existing nanostructures would commence at the edge-sites, where bonding is most fragile, and gradually work inward. Oxidation using a $\mathrm{O}_{2}$ plasma treatment (PT) was employed. Figure 6 shows a side-by-side comparison of the sample (a) sulfided at $413^{\circ} \mathrm{C}$ and the same sample then (b) oxidized with a plasma treatment. Examination of the two images suggests that in Figure $6 \mathrm{a}$ the $\mathrm{WS}_{2}$ edge-structures formed facets with well-defined edges; whereas, in Figure 6b the subsequent plasma treatment resulted in several discontinuities in the edge-structure. Figure $6 \mathrm{c}$ shows a quantitative account of these findings, where $\mathrm{P}$ is plotted versus $\mathrm{A}$ for the two samples. Based on the different trajectories of the scatter, it can be surmised that the PT was effective at creating more undercoordinated edge-sites. This result supports the assumption that subjecting metal sulfides to PT, resulting in edge modifications similar to the Ni-promoted samples, could lead to performance gains similar to that of the promoted catalyst.

\section{Conclusions}

The findings in this article demonstrate important advances in nanomaterial processing and characterization. Multiple methods were shown to produce quantifiable modifications of nanomaterials at atomic-length scales. Documentation of these subtleties mandates that analyses be carried out with a probecorrected field-emission STEM at a minimum. Collectively the data show how previously unperceivable, atomic-scale changes can lead to dramatic large-scale changes in catalyst performance. Furthermore we show several examples of nanostructure modifications that previously would have been unobserved and undocumented if not for advances in electron microscopy. 

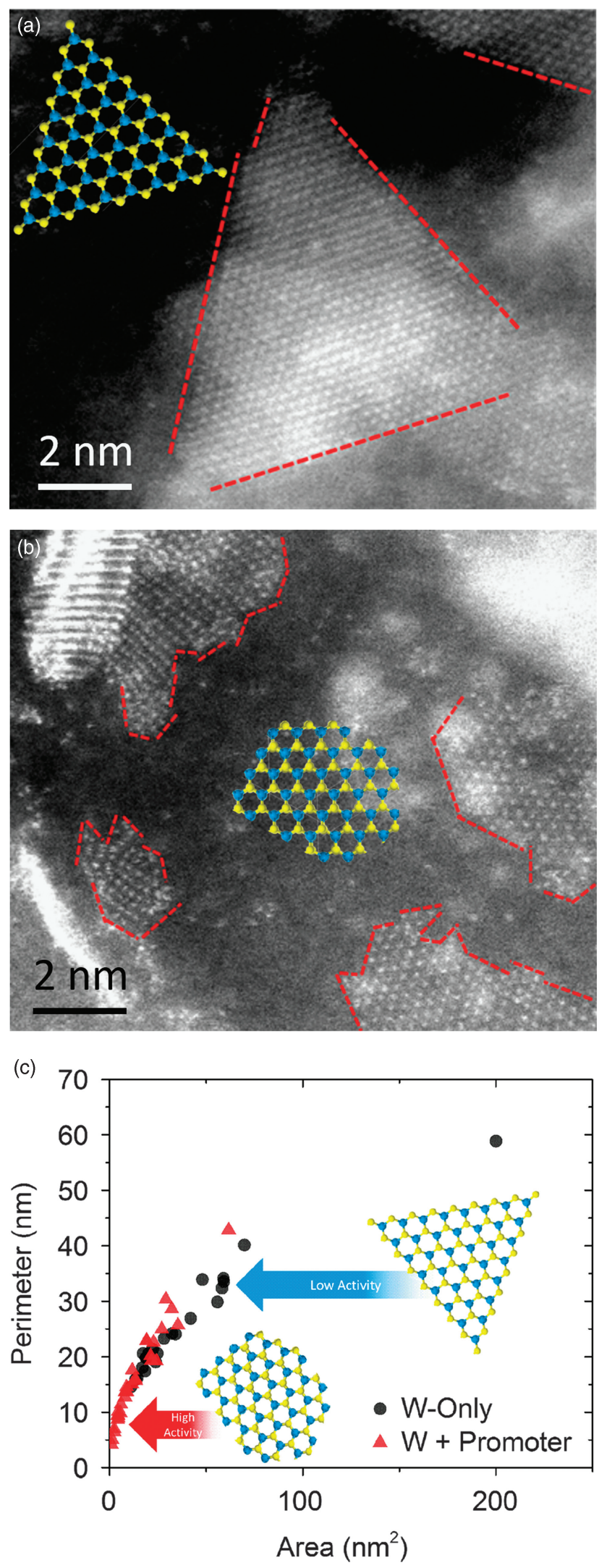

Figure 5: Atomic-resolution HAADF STEM images of (a) $\mathrm{WS}_{2}$ and (b) $\mathrm{Ni}$-promoted $\mathrm{WS}_{2}$ nanostructures. Contrast is from $\mathrm{W}$ atoms only. Red dashed lines guide the eye to the edges of the sheet-like structures. (c) Plots of P/A ratios for the two samples. Blue and yellow DFT models of the metal sulphide structures indicate differences in edge-structure.
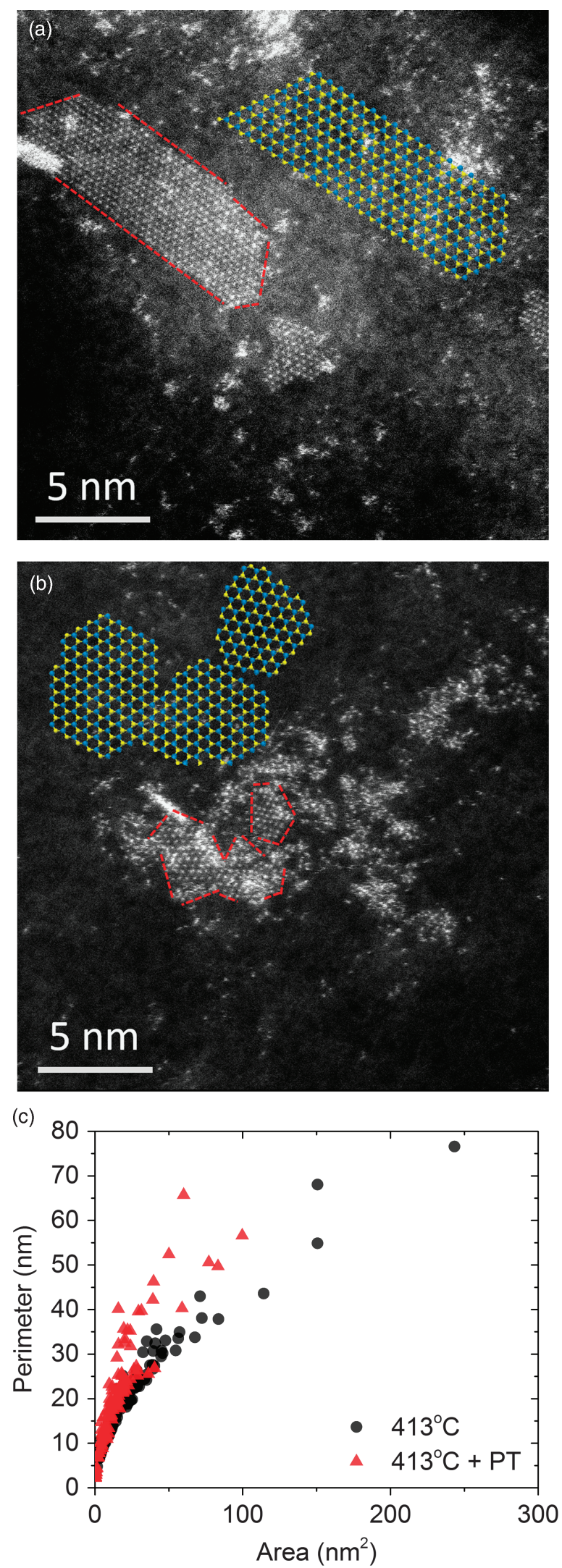

Figure 6: Atomic-resolution HAADF STEM images of $\mathrm{WS}_{2}$ nanostructures (a) sulfided at $413^{\circ} \mathrm{C}$ and (b) sulfided at $413^{\circ} \mathrm{C}+\mathrm{O}_{2}$ plasma treatment. Contrast is from $W$ atoms only. Red dashed lines guide the eye to the edges of the sheet-like structures. (c) Plots of P/A ratios for the two samples. Blue and yellow insets are DFT models of the metal sulfide, which indicate differences in edge-structure. 


\section{Acknowlegments}

Research was supported in part by Oak Ridge National Laboratory's Center for Nanophase Materials Sciences, which is sponsored by the Scientific User Facilities Division, Office of Basic Energy Sciences, U.S. Department of Energy.

\section{References}

[1] M Varela et al., Annu Rev Mater Res 35 (2005) 539-69.

[2] G Schmid, Nanoparticles: From Theory To Application. Wiley-VCH Verlag GmbH \& Co, Weinheim, 2004.

[3] RW Siegel, Nanostructure Science And Technology: R\&D Status and Trends In Nanoparticles, Nanostructured Materials and Nanodevices. Springer, Netherlands, 1999.

[4] RA Mayers, Handbook Of Petroleum Refining Processes, 3rd Ed, McGraw-Hill, New York, 2003.

[5] JWA Sachtler and GA Somorjai, J Catal 81 (1983) 77-94.

[6] PK Doolin, Catalytic Naphtha Reforming, 2nd Ed, Marcel Dekker, Inc, New York, 2004, pp 444-45.

[7] X Xu et al., Nanotoday 9(5) (2014) 604-30.

[8] ML Bricker et al., Hydrocracking Industry Today: Development Of New Catalysts With The Assistance Of Advanced Characterization, $244^{\text {th }}$ ACS National Meeting, Philadelphia, PA, 2012.

[9] M Girleanu et al., ChemCatChem 6 (2014) 1594-98.

[10] SD Kelly et al., J Catal 263 (2009) 16-33.

[11] Y Zhu et al., Angewandte Chemie Int Ed 53 (2014) 10723-27.

\section{PELCO'silicon Nitride} \& Silicon Dioxide Membranes

\section{Next Generation SiN TEM Support Films}

- Robust and clean 8, 15, 50 and $200 \mathrm{~nm}$ SiN substrates

- ø3.0mm frame

- EasyGrip ${ }^{\text {TM }}$ edges

- Free from debris

- Super flat 8,15, and 40nm silicon dioxide substrates

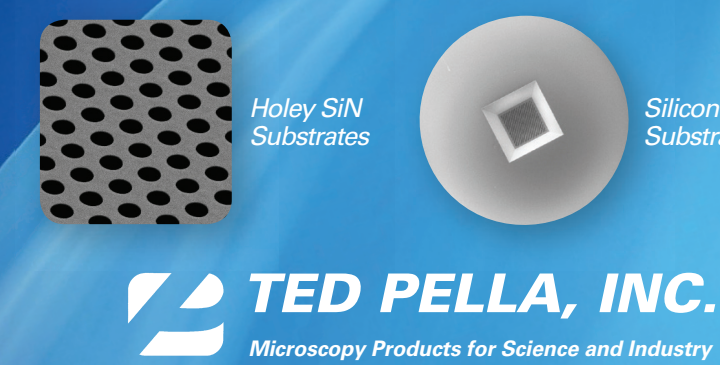

www.tedpella.com sales@tedpella.com 800.237.3526 MT

\section{$\infty \Delta$ \\ 1 TO 50 MEGAPIXELS live and slow scan \\ MAGNIFICATION FACTOR OF 1 on bottom mounted cameras \\ DIFFRACTION BEAM STOP on side mounted cameras}
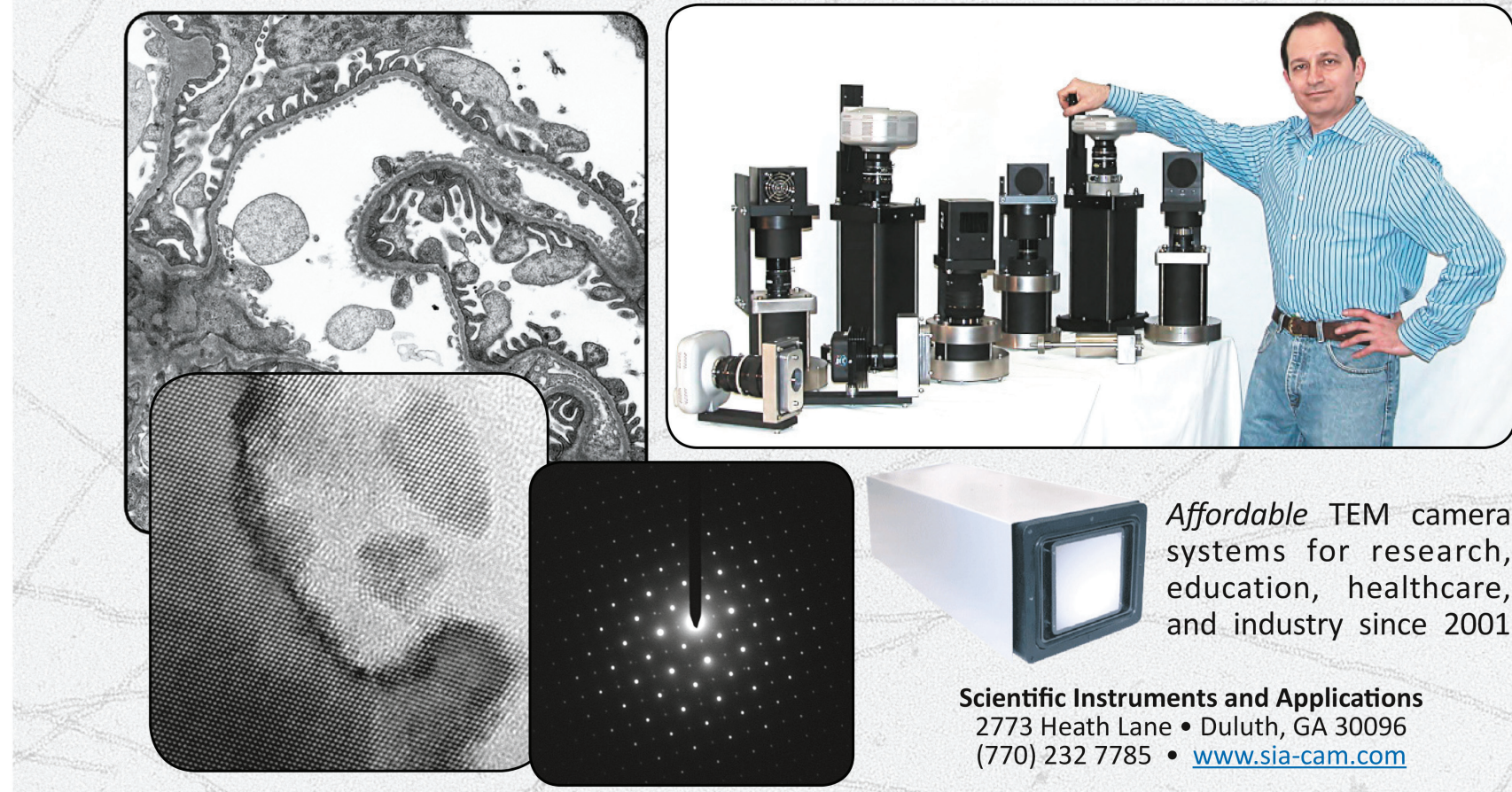

Affordable TEM camera systems for research, education, healthcare, and industry since 2001

Scientific Instruments and Applications

2773 Heath Lane • Duluth, GA 30096

(770) $2327785 \cdot$ www.sia-cam.com 

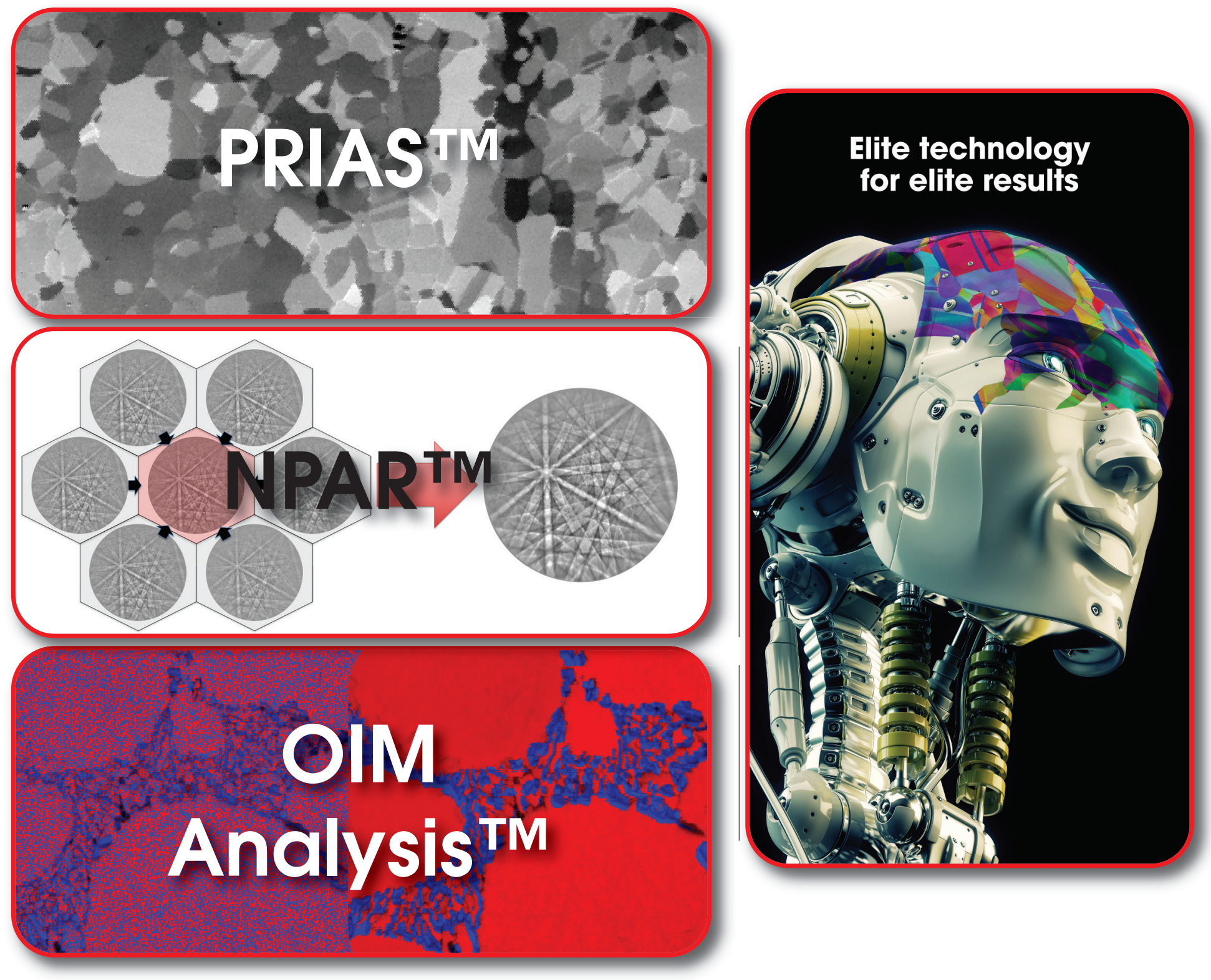

\section{High quality data collection is just the start.....}

- $\quad$ PRIAS ${ }^{\text {TM }}$ - an innovative approach for enhancing EBSD sample imaging

- $\quad$ NPAR $^{\text {TM }}$ - a unique way to improve EBSD indexing

- OIM Analysis ${ }^{\text {TM }}$ - a comprehensive toolbox for analysis of EBSD data

- $\quad$ EBSD detector family - with results without compromise for all applications 